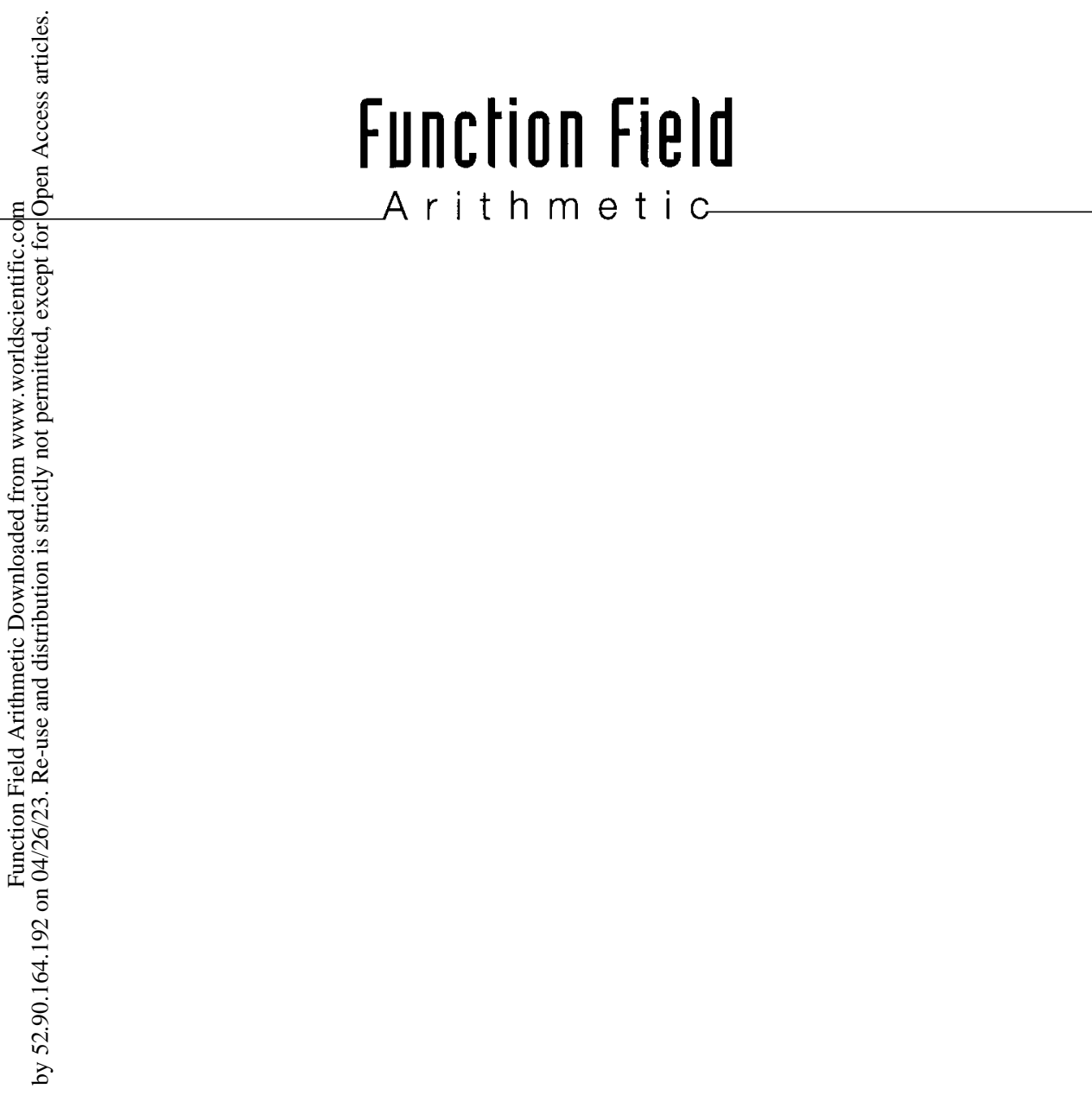


This page intentionally left blank 


\section{Function Field}

\section{A rith $\mathrm{met}$ i $\mathrm{c}$}

by

Dinesh S. Thakur

University of Arizona, USA

\section{Noworld Scientific}




\section{Published by}

World Scientific Publishing Co. Pte. Ltd.

5 Toh Tuck Link, Singapore 596224

USA office: Suite 202, 1060 Main Street, River Edge, NJ 07661

UK office: 57 Shelton Street, Covent Garden, London WC2H 9HE

\section{Library of Congress Cataloging-in-Publication Data}

Thakur, Dinesh S., 1961 -

Function field arithmetic / by Dinesh S. Thakur.

p. $\mathrm{cm}$.

Includes bibliographical references and index.

ISBN 981-238-839-7 (alk. paper)

1. Fields, Algebraic. 2. Arithmetic functions. 3. Drinfeld modules. I. Title.

QA247.T53 2004

512'3--dc22

2004041975

\section{British Library Cataloguing-in-Publication Data}

A catalogue record for this book is available from the British Library.

Copyright (C) 2004 by World Scientific Publishing Co. Pte. Ltd.

All rights reserved. This book, or parts thereof, may not be reproduced in any form or by any means, electronic or mechanical, including photocopying, recording or any information storage and retrieval system now known or to be invented, without written permission from the Publisher.

For photocopying of material in this volume, please pay a copying fee through the Copyright Clearance Center, Inc., 222 Rosewood Drive, Danvers, MA 01923, USA. In this case permission to photocopy is not required from the publisher. 


\section{Dedicated to the fond memories of Bhaikaka, to Maiatte, Bhai, Aai; and to my other teachers, family and friends}


This page intentionally left blank 


\section{Preface}

This book, written for those with appreciation for number theory and geometry, gives an exposition of function field arithmetic with emphasis on recent developments having to do with Drinfeld modules, arithmetic of special values of transcendental functions, diophantine approximation and on related interesting open problems. The subject matter is a beautiful blend of arithmetic, algebra, geometry and analysis. We assume a basic background in algebraic number theory and geometry at least at the level of curves.

Number theorists study number fields and function fields together because of various analogies between them in spite of some important differences. Interesting arithmetic of function fields was developed by Kronecker, Dedekind, Weber, Emil Artin, F. K. Schmidt, Witt, Hasse, Weil, Chevalley, Lang, Tate, Serre, Shafarevich, Manin, Parshin, Iwasawa, Grothendieck, Deligne, Arakelov, and on the Drinfeld module side that we write more about by Carlitz, Wade, Wagner, Hayes, Drinfeld, Deligne, Goss, Gekeler, Galovich, Rosen, Jing Yu, Takahashi, Stuhler, Gross, Anderson to name just a few early contributors.

New analogies understood by Carlitz, Drinfeld, Goss, Anderson etc. opened up new territories. The subject is now a mixture of well developed areas, some where analogs to conjectures in the number field case are proved and well-understood, so that there are attempts to bring these ideas $\vec{\sigma}$ in the number fields case in various ways, and some other promising areas, largely unexplored, where the number field case is well-understood and the function field case goes against the naive analogies and is not even conjecturally understood. The good examples of the first type are Riemann hypothesis, Iwasawa theory, Birch and Swinnerton-Dyer conjectures and Langlands conjectures, transcendence theory and special values of Gamma 
functions, whereas the special values of Zeta functions, Diophantine approximation are of the second type.

Several analogies work in $\mathbb{F}_{q}[t]$ case, but separate to quite different phenomena in general, unlike earlier theories of phenomena which worked quite smoothly in general, if only with minor changes. This split-up helps us focus on relevant issues. Out of many equivalent ways to look at a particular $\frac{\ddot{0}}{O}$ classical concept, the way which translates well in the function field case may be thought of as a more fundamental one. Hence the analogs help to sharpen our understanding of classical concepts. Further, as we will see, the function field arithmetic is in interesting hybrid analogies with number field case, and its $p$-adic and $q$-analogs.

Here is Andre Weil ( [Wei79, Vol. II, p. 408] as translated by Mazur) talking about the magic and importance of analogies in mathematics:

Nothing is more fruitful - all mathematicians know it - than those obscure analogies, those disturbing reflections of one theory on another; those furtive caresses, those inexplicable discords; nothing also gives more pleasure to the researcher.

Weil goes on talking about what happens when everything is understood. Fortunately, we are far from that stage here, and the exciting exploration goes on.

In contrast to the earlier function field situation, many areas such as special values of zeta functions, diophantine approximation are not even conjecturally understood. But concrete results that have been obtained suggest a beautiful interesting structures, only partially revealed. Things which do not seem to work as expected are the most interesting things.

In this book, I have tried to tell this interesting story to my fellow students of Mathematics, hoping to share the excitement and the enjoyment I felt working on it. The outline of the book can be best understood by taking a look at the index and introductions to the chapters. One impor$\sigma$ tant theme is the algebraic incarnations of gamma and zeta values, and to related special functions on product of curves which govern the arithmetic \& of gamma, zeta values, theta functions and Gauss sums.

ก For me, it was quite a tough choice between using this occasion to write this book for setting things up in more generality for future reference or going for examples and explanations in the simple motivating cases leaving out the technicalities and the generalizations. Both approaches have their virtues. For the better or for the worse, I have finally opted for the second option. It is indeed true that many of the interesting results and mysteries already show up in the simplest cases. I have not tried to be in the most 
general setting possible or to give complete proofs each time. I hope that the sketch or sometimes even the catch-words will provide some insight. I have often sacrificed efficiency by dealing with special cases first and spelling out easy consequences. Especially we do $\mathbb{F}_{q}[t]$ case quite often: Many times it can be done easily explicitly with easier different proofs, and also because many times answers are quite different in that case, many analogies working simultaneously, whereas they break up into different analogies for other $A$ in higher genus.

Simplest way to proceed is usually not the historical one: false attempts, which add to understanding nonetheless, are ignored, and tricks, original ideas or important remarks, at the time, eventually become part of the standard technique. And a lot looks easier and straightforward in retrospect. So in writing a book many times giving proper attributions is hard and referring to the original articles is the best source. I have followed the frequent practice in books of mentioning some relevant references in the notes, rather than in each place as required in research articles. So for attributions original sources need to be consulted. Many times (especially for facts mentioned in passing) I have given only the most convenient reference where you can find the original sources. There is also an extensive bibliography in the book by Goss.

While making comparisons with the literature, note that the notation as well as the normalizations differ in original sources. We use the same notation and name for analogous objects in different context: For example, gamma function $\Gamma$ stands for the classical gamma function as well as one of the Gamma functions for function fields or its special case for say $\mathbb{F}_{q}[t]$. The role should be clear from the context and is usually specified. The precise bookkeeping of the notations would have made it harder to read.

This is just a tour of some topics I enjoyed learning and working on, and is by no means exhaustive treatment. Focusing on certain areas and applications, we have omitted or given much shorter treatment (just sketching some ideas and results) than deserved to many topics, such as the moduli questions and topics related to Langlands conjectures, i.e., the original motivations of Drinfeld (see papers of Drinfeld, Deligne-Husemoller, Laumon, Lafforgue and books by Laumon, Gekeler), modular forms (see book by Gekeler), Artin-Hasse-Weil theory and Diophantine geometry (treated in many books).

Only regret I have is that I had to resist the urge to pursue in more detail many things which came up, because of time constraints, and page-limit. I will be very glad if the reader (or readers, let us be optimistic) takes trouble 
to comment, suggest improvements, points out errors and references etc. I plan to provide updates on the web page.

I thank many people and institutes for their help: Institute for Advanced study, where the writing started and University of Arizona, Tata Institute of Fundamental research and Bhaskaracharya Pratishthan where it continued; my colleagues at these and other places; National Science $\frac{0}{U}$ Foundation, National Security Agency and U. S. Civilian Research and Development Foundation for providing financial support during this period; collaborators Jean Paul Allouche, Greg Anderson, Robert Beals, Minhyong Kim, Felipe Voloch as well as other friends in this area of mathematics such as David Hayes, Ernst Gekeler, Jing Yu, Michael Rosen, Bjorn Poonen, Yuchiro Taguchi, Dale Brownawell, Anatoly Kochubei, Matt Papanikolas.

With his basic contributions as well as tremendous enthusiasm and energy, David Goss has been one of the main leaders and the driving force of the field in the last many years. When I was hesitating to reply positively to the invitation to write this book, as David Goss' book had just been published, David encouraged me to write saying that it would certainly be better to hear different perspectives. I thank him and Greg Anderson for their generosity over the years. I could always count on my friends Greg, David, Ernst, Takahiro Shiota and Jeremy Teitelbaum over the years for trying to answer anything that I would ask.

I dedicate this book to Bhaikaka's memory. He retained informality of conversation in his writings. This is sometimes difficult while communicating mathematical ideas, but I have tried my best, given the constraints, and I would like to think that he would have approved of at least the effort.

This book is also dedicated to my teachers, family and friends, fortunately too many to be listed here. The three categories are also well-mixed. But I must mention John Tate, Barry Mazur, Pierre Deligne and Greg Anderson from whom I learned a lot (though the content and the crude style ऽ of this book might prove exactly the opposite!). On the non-mathematical tं front, I owe a lot to Bhaikaka, Maiatte, Bhai, Aai, Satish, Shubha, Umesh; and to Jyoti and Ashutosh, who kept on nagging me about finishing the in book and at the same time provided welcome distraction. ถి

December 2003

Dinesh S. Thakur 


\section{Contents}

Number fields and Function fields 1

1.1 Global fields: Basic analogies and contrasts . . . . . . . . 1

1.2 Genus and Riemann-Roch theorem . . . . . . . . . . . 9

1.3 Zeta function and class group . . . . . . . . . . . . . . . 13

1.4 Class field theory and Galois group . . . . . . . . . 20

$\begin{array}{ll}\text { Drinfeld modules } & 31\end{array}$

2.1 Carlitz module and related arithmetical objects . . . . . . . 31

2.2 Drinfeld modules: Basic definitions . . . . . . . . . . . . . . 34

2.3 Torsion points . . . . . . . . . . . . . . . . . 39

2.4 Analytic theory . . . . . . . . . . . . . . . 41

2.5 Explicit calculations for Carlitz module . . . . . . . . . 44

2.6 Reductions . . . . . . . . . . . . . . . . . . . . . . . . . . . . . . . . .

2.7 Endomorphisms . . . . . . . . . . . . . 50

2.8 Field of definition . . . . . . . . . . . . . 52

2.9 Points on Drinfeld modules . . . . . . . . . . . . 53

2.10 Adjoints and duality . . . . . . . . . . . . . 54

2.11 Useful tools in non-archimedean or finite field setting . . . . 56

(a) Properties of $k\{\tau\} \ldots \ldots 57$

(b) Moore determinant .......... 58

(c) $\quad$-resultants .............. 58

(d) Non-archimedean calculus . . . . . 59

(e) Dwork's trace formula ....... 61 
3. Explicit class field theory

3.1 Torsion of rank one Drinfeld modules . . . . . . . . . . . . 64

3.2 Sign normalization of the top coefficient . . . . . . . . 68

3.3 Normalizing Field as a class field . . . . . . . . . . . . 70

3.4 Smallest field of definition as a class field . . . . . . . . 73

3.5 Ring of definition . . . . . . . . . . . . . . . . 74

3.6 Cyclotomic fields . . . . . . . . . . . . . . 76

3.7 Moduli approach . . . . . . . . . . . . . . . . 77

3.8 Summary . . . . . . . . . . . . . . . . . . . . . . 79

3.9 Maximal abelian extension . . . . . . . . . . . . . . 80

3.10 Cyclotomic theory of $\mathbb{F}_{q}[t] \ldots \ldots \ldots$. . . . . . . 82

3.11 Cyclotomic units and conjectures of Brumer and Stark . . . 83

3.12 Some contrasts and open questions . . . . . . . . 85

$\begin{array}{ll}\text { Gauss sums and Gamma functions } & 87\end{array}$

4.1 Gauss and Jacobi sums: Definitions . . . . . . . . . . 88

4.2 Gauss and Jacobi sums: $\mathbb{F}_{q}[t]$ case . . . . . . . . . . . 91

4.3 Gauss and Jacobi sums: General $A \ldots \ldots . . \ldots 9$

4.4 Sign of Gauss sums for $\mathbb{F}_{q}[t] \ldots \ldots 9$

4.5 Arithmetic Factorial and Gamma: Definitions . . . . . . . . 101

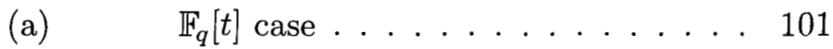

(b) General $A \ldots \ldots . \ldots 103$

4.6 Functional equations in arithmetic case . . . . . . . . 105

4.7 Special values for arithmetic $\Gamma_{\infty} \ldots \ldots \ldots . \ldots 109$

(a) Periods: $\mathbb{F}_{q}[t]$ case . . . . . . . 109

(b) Periods: General $A \ldots \ldots 110$

4.8 Special values of arithmetic $\Gamma_{v} \ldots \ldots \ldots$. . . . . . . 113

(a) $\quad \mathbb{F}_{q}[t]$ case: Analog of Gross-Koblitz . 113

(b) General $A \ldots \ldots . \ldots 114$

4.9 Geometric Factorial and Gamma: Definitions . . . . . . . 116

4.10 Functional equations in geometric case . . . . . . . . . 118

4.11 Special values of geometric $\Gamma$ and $\Gamma_{v}: \mathbb{F}_{q}[t]$ case . . . . . 122

4.12 Comparisons and uniform framework . . . . . . . . . . . . . 124

4.13 More analogies for $\mathbb{F}_{q}[t]$ : Divisibilities . . . . . . . . . . 130

4.14 Binomial coefficients . . . . . . . . . . . . . . . . . . 132

(a) Binomial coefficients as nice basis . . 134

(b) Difference and differentiation operators 136

4.15 Relations between the two notions of binomials . . . . . . 138 
4.16 Bernoulli numbers and polynomials . . . . . . . . . . . . . 142

4.17 Note on finite differences and $q$-analogs $\ldots \ldots$. . . . . . 149

5. Zeta functions 153

5.1 Zeta values at integers: Definitions . . . . . . . . . . 154

5.2 Values at positive integers . . . . . . . . . . 158

5.3 Values at non-positive integers . . . . . . . . . . . 162

5.4 Multiplicities of trivial zeros . . . . . . . . . . . . 166

5.5 Zeta function interpolation on character space . . . . . . 170

(a) $\infty$-adic interpolation $\ldots \ldots 170$

(b) $\wp$ adic interpolation . . . . . . 173

5.6 Power sums . . . . . . . . . . . . . . . . . 174

5.7 Zeta measure . . . . . . . . . . . . . 176

5.8 Zero distribution . . . . . . . . . . . . . . . . . . 178

5.9 Low values and multi-logarithms . . . . . . . . . . . 182

5.10 Multizeta values . . . . . . . . . . . . . . . . 185

(a) Complex valued multizeta . . . . 186

(b) Finite characteristic variants . . . . 187

(c) Interpolations . . . . . . . . . 195

5.11 Analytic properties of zeta and Fredholm determinant . . . 197

5.12 Note on classical interpolations . . . . . . . . . . . . . . . 199

6. Higher rank theory 205

6.1 Elliptic modules . . . . . . . . . . . . . . . 205

6.2 Modular forms . . . . . . . . . . . . . . . . . 209

6.3 Galois representations . . . . . . . . . . . . . . . . . . . . . . . . . . . . . 213

6.4 DeRham Cohomology . . . . . . . . . . . . . 218

(a) Elliptic curves case: Motivation . . . 219

(b) Drinfeld modules case . . . . . . . 221

6.5 Hypergeometric functions . . . . . . . . . . . . . 226

(a) The first analog . . . . . . . . . 227

(b) The second analog ....... 233

7. Higher dimensions and geometric tools 237

$7.1 t$-modules and $t$-motives . . . . . . . . . . . 239

7.2 Torsion . . . . . . . . . . . . . . . . . . 244

7.3 Purity . . . . . . . . . . . . . . . . . 244

7.4 Exponential, period lattice and uniformizability . . . . . 247 
7.5 Cohomology realizations . . . . . . . . . . . . . 253

7.6 Example: Carlitz-Tate twist $C^{\otimes n} \ldots \ldots . \ldots . . . \ldots 254$

7.7 Drinfeld dictionary in the simplest case . . . . . . . 256

7.8 Krichever/Drinfeld dictionary in more generality . . . . . 260

8. Applications to Gauss sums, Gamma and Zeta values 265

$8.1 C^{\otimes n}$ and $\zeta(n) \ldots \ldots \ldots \ldots \ldots \ldots$

8.2 Shtuka and Jacobi sums . . . . . . . . . . . . . . . 267

(a) Gauss sums and Theta divisor . . . 2 269

(b) Examples and applications . . . . . 269

(c) The case $g=d_{\infty}=1 \ldots 271$

8.3 Another Gamma function . . . . . . . . . . . . . . . . 273

(a) Analog of Gross-Koblitz . . . . . 276

(b) Interpolation at $\infty$ for new Gamma . 276

8.4 Fermat motives and Solitons . . . . . . . . . . . . 280

8.5 Another approach to solitons . . . . . . . . . . . 286

8.6 Analog of Gross-Koblitz for Geometric Gamma: $\mathbb{F}_{q}[t]$ case . 290

8.7 What is known or expected in general case? . . . . . . . . . 290

8.8 Gamma values to Periods connection via solitons: Sketch . 293

8.9 Log-algebraicity, Cyclotomic module and Vandiver conjecture 295

8.10 Explicit Log-Algebraicity formulas . . . . . . . . . . . . 298

9. Diophantine approximation 303

9.1 Approximation exponents . . . . . . . . . . . . . . . 304

9.2 Good approximations: Continued fractions . . . . . . 306

9.3 Range of exponents : Frobenius . . . . . . . . . . . . . . 312

9.4 Range of exponents: Differentiation . . . . . . . . . . . . . 319

9.5 Connection with deformation theory . . . . . . . . . 322

(a) Height inequalities for algebraic points 323

(b) Exponent hierarchy . . . . . . 324

(c) Approximation by algebraic functions 325

9.6 Note on connection with Diophantine equations . . . . . . 326

10. Transcendence results $\quad 329$

10.1 Approximation techniques and irrationality . . . . . . . 330

10.2 Transcendence results on Drinfeld modules . . . . . . . . 331

10.3 Application to Zeta and Gamma values . . . . . . . . . . 334

10.4 Transcendence results in higher dimensions . . . . . . . 335 
10.5 Application to Zeta and Gamma values . . . . . . . . . 337

11. Automata and algebraicity: Applications 341

11.1 Automata and algebraicity . . . . . . . . . . . . . . . . . . . . . 341

11.2 Some useful automata tools . . . . . . . . . . . . . . 347

11.3 Applications to transcendence of gamma values and monomials 348

11.4 Applications to transcendence: periods and modular functions 353

11.5 Classifying finite characteristic numbers . . . . . . . . . 355

11.6 Computational classes and basic tools . . . . . . . . . . . . 357

11.7 Algebraic properties of computational classes . . . . . . . 360

11.8 Applications to refined transcendence . . . . . . . . . . 364

Note on the Notation 371

$\begin{array}{ll}\text { Bibliography } & 373\end{array}$ 